\title{
Découverte d'une nouvelle métalloendopeptidase de la surface cellulaire impliquée dans les métastases
}

\begin{abstract}
Depuis la découverte des collagénases, il y a de cela environ trente ans, on pense que certaines protéases jouent un rôle important dans la dégradation de la matrice extracellulaire. Ce phénomène est observé lors de la morphogenèse, la réparation des os et du cartilage, la guérison des blessures, l'angiogenèse et la migration des neutrophiles [1, 2]. Ces mêmes protéases pourraient être aussi impliquées dans des phénomènes pathologiques tels que l'inflammation et l'invasion des tissus par des métastases tumorales [2-4]. Des études in vitro suggèrent que les processus de dégradation de la matrice extracellulaire qui accompagnent les métastases sont confinés à des régions particulières de la surface cellulaire où ces protéases occuperaient une position stratégique pour aider les cellules à se frayer un chemin au travers des tissus [5]. La dégradation de la matrice extracellulaire est accomplie par des protéases intrinsèques de la membrane, ou encore par des protéases solubles sécrétées sous forme de précurseurs inactifs qui seraient activés par l'action d'autres protéases à la surfa-
\end{abstract} ce cellulaire [3].

La plupart des protéases sécrétées qui ont été décrites jusqu'à présent sont assez abondantes. Leur forme soluble majoritaire est toutefois présente sous forme de zymogène inactif, alors que la forme active se retrouve, quant à elle, associée à la surface cellulaire. On peut donc identifier quatre acteurs principaux qui jouent un rôle dans la modulation de l'activité protéolytique associée à la surface cellulaire : (1) les protéases solubles elles-mêmes; (2) les récepteurs qui les retiennent à la surface cellulaire; (3) les enzymes protéolytiques impliquées dans la matu- des inhibiteurs chargés de moduler l'activité protéolytique après activation.

Compte tenu de la grande diversité des substrats sur lesquels agissent ces protéases et de la résistance à la protéolyse des composants de la matrice extracellulaire, il n'est pas surprenant de retrouver une grande variété d'enzymes protéolytiques qui collaborent à la dégradation de cette matrice. La plupart des enzymes protéolytiques appartiennent à l'une ou l'autre de deux grandes familles : les sérine protéases, en particulier le système activateur du plasminogène/plasmine [6] et les métalloprotéases de la matrice (MMP) [7, 8]. On a identifié au moins neuf MMP différentes ; l'une d'entre elles, la gélatinase A est responsable de la dégradation du collagène de types IV et $\mathrm{V}$, de l'élastine et de la laminine [7]. On retrouve souvent des quantités élevées de gélatinase A dans les cellules stromales des tumeurs malignes [9]. Comme bien d'autres MMP, la gélatinase A est sécrétée sous forme de proenzyme inactive, mais la progression tumorale s'accompagne d'une augmentation de la quantité de forme active de cette protéase [10] dont l'activité catalytique doit ainsi être contrôlée de façon très stricte. Cette régulation a lieu à la surface cellulaire et donne naissance à une enzyme active qui reste associée à la membrane plasmique en se liant à un récepteur dont la nature est inconnue [11]. Un grand pas dans la compréhension de l'activation de la gélatinase A vient d'être accompli par l'équipe de Sato (Kanasawa, Japon) [12] qui a identifié une métalloprotéase de la surface cellulaire responsable de la maturation de la progélatinase $\mathrm{A}$ en gélatinase $\mathrm{A}$ active. Cette enzyme pourrait donc jouer un rôle clef dans la cascade d'événements impliqués dans le remodelage de la matrice extracellulaire.

Sato et al. ont utilisé la technique de PCR pour amplifier, dans une banque d'ADNc de placenta humain, un fragment d'ADNc ayant une similarité de séquence avec d'autres membres connus de la famille des métalloprotéases (stromélysine- 3 , stromélysine-1, collagénase, gélatinase A et gélatinase $B$ ). La protéine codée par cet ADNc se distingue des autres membres de la famille par la présence d'une séquence hydrophobe aux caractéristiques de segment transmembranaire. Cette nouvelle protéase devrait donc être une protéine intrinsèque de la membrane ; elle a été effectivement retrouvée à la surface cellulaire des cellules après transfection. Pour la distinguer des autres MMP solubles, Sato et al. l'ont donc appelée MT-MMP (membrane-type MMP). Exprimée dans des lignées cellulaires sécrétant de la progélatinase $\mathrm{A}$ ou B, l'enzyme s'est révélée capable de transformer les formes précurseurs en produits comparables aux enzymes actives retrouvées dans les cellules tumorales. De plus, Sato et al. ont pu démontrer la présence de cette enzyme dans des carcinomes pulmonaires où on la retrouve en plus grande quantité que dans le poumon normal. Les cellules NIH 3T3 qui expriment à la fois la progélatinase A endogène et la MT-MMP recombinante ont un pouvoir invasif plus important que les cellules n'exprimant pas l'enzyme ou exprimant une forme tronquée et soluble de celle-ci. Cela conforte l'hypothèse que cette MT-MMP pourrait être impliquée dans la formation des métastases.

Cet article soulève aussi d'autres questions importantes. L'enzyme est 
également exprimée dans le placenta et dans le rein. Quel est son rôle dans ces tissus? Comme d'autres membres de la même famille, l'enzyme membranaire possède une "prorégion " dont le clivage est peut-être nécessaire pour conférer une activité catalytique. Est-ce vraiment le cas, et quel en serait le mécanisme? Cette enzyme agit-elle sur d'autres substrats que la progélatinase A ? Y a-t-il d'autres membres de la même famille à la surface cellulaire pour activer d'autres MMP? Ce travail ouvre certainement la voie à d'autres travaux dont les résultats pourraient avoir des conséquences importantes dans la thérapie du cancer.

P.C.
I. Birkedal-I lansen II, Werb Z. Welgus IIC; Van Wart HF. Matrix metalloproteinases and inhibitors Proceedings of the Matrix Metalloproteinase (isnference held at Sandestin Beach, FL, Sept. 11-15, 1989. Jena: ( Bustav Fisher Verlag, 1992: 1-50)1.

2. Vandenbunder B, Faleur V' Wernert N, Stéhelin D). Analyse moléculaire de l'angiogenèse tumorale. méderine/sriences $1994: 10: 516-27$.

3. Woll (; lefebve (), Rouver N. (henard MP Bellocq JP, Rio MC: Chambon P. Basset P. Pro teases dorigine stromale et progression tumorale. médecine/sciences $1994: 10: 507-15$

4. Alexander (C.M, Werb \%. Extracellular matrix degradation. In : Hav F.l). ed. C.ell biology of extra cellular matrix. New York: Plenum Press. 1991 $255-304$.

5. Chen WT. Membrane proteases : roles in tissue remodeling and tumor invasion. (iur ()p Cell Biol 1992; $4: 8(1)-9$.

6. Vassalli JD, Sappino AP, Belin D. The plasminogen activator/plasmin system. I C:lin Invest $1991: 88: 1067-72$
7. Matrisian I.M. The matrix-degrading metalloproteinases. Bioessays 1992 : $14: 455-63.3$.

8 . Kleiner D):. Stetler-Stevenson (;. Structural biochemistry and activation of matrix metalloproteases. (iur Of) (:ell Biol 1993; 5 : 891-7.

9. Pyke C, Rallkiaer F., Tivggaason $k$, Dano $k$ Messenger RN I for two type IV collagenases is located in stromal cells in human colon cancer. Am / Pathol l993: 142 : 359-(65.

10. Brown Pl), Bloxidge RF, Stuart NS, (jatter Ki(: Carmichael J. Association between expression of activated 72 -kilodalton gelatinase and tumor spread in non-small-cell lung carcinoma. / Vatl Ciucer Inst 1993; $85: 574-8$.

II. Fmonard IIP. Remacle AC, Nod AC: (irimatud JA, Steter-Stevenson WC, Foidant J.M. Tumor cell surlace-associated binding site for the M(r) 72,000 type IV collagenase. Cancer Res $1992 ; 52: 58.15-8$.

12. Sato H. Takino T, ()kada Y, (aa) I Shinagawa A. Yamamoto li. Sciki M. A matrix metalloproteinase expressed on the surface of invasive tumour cells. Nature 1994; 370 : $61-5.5$.

\section{BRÈVES}

Rôle de la protéine $R b$ et de p53 dans l'équilibre entre prolifération et apoptose de la rétine et du cristallin. Les gènes $R b$ et $p 53$ sont considérés comme des antioncogènes. $p 53$ joue également un rôle très important dans le contrôle de l'apoptose [1]. Des souris transgéniques exprimant l'antigène $T$ du virus SV40 dans la rétine, sous le contrôle du promoteur codant pour l'IRBP (interstitial retinal-binding pro(ein), développent un rétinoblastome. Cependant, l'antigène $T$ est capable de se lier tout à la fois au produit du gène $R b$ et à la protéine p53, si bien que le rôle respectif de l'inactivation de ces deux protéines dans l'apparition de la tumeur ne peut être établi. Howes, el al. (San Antonio, TX, et Madison, W'I, USA) ont résolu ce problème de manière élégante. Ils ont construit des souris transgéniques exprimant le gène $E 7$ du papillomavirus humain de type 16 (HPV-16 E7) sous le contrôle du promoteur du gène $I R B P$. E7 peut complexer la protéine $\mathrm{Rb}$, mais pas p53. Les animaux transgéniques ne développent pas de cancers mais, au contraire, une dégénérescence rétinienne en rapport avec une apoptose des photorécepteurs
[2]. En revanche, le même transgène chez des souris dont les deux allèles $p 53$ ont été mutés par recombinaison homologue induit l'apparition d'une tumeur rétinienne. On peut interpréter ces résultats en proposant que l'inactivation de $R b$ par la protéine E7 entraîne une stimulation du cycle cellulaire, donc un signal mitotique, non coordomné : dans ces conditions, ce gardien de la prolifération cellulaire qu'est p53 entraîne l'apoptose [1]. Lorsque le gardien lui-même est absent, la stimulation mitotique aboutit à la formation d'une tumeur. Pan et Griep, de Madison (WI, USA) ont étudié l'influence des mêmes antioncogènes dans le cristallin de l'œil, en utilisant une stratégie légèrement différente. Des souris transgéniques synthétisant les protéines E6 ou E7 de papillomavirus humain sous le contrôle du promoteur du gène de l' $\alpha$ cristalline $(\alpha A)$ ont été créées. Les souris simples transgéniques ont, dans les deux cas, une cataracte dont les caractéristiques sont cependant légèrement différentes en ce que les souris $\alpha$ AE6 n'ont pas de microphtalmie alors que ce signe existe chez les souris aAE7. A l'examen histologique, les animaux
aAE6 possèdent des fibres cristalliniennes qui demeurent nucléées, ce qui suggère que l'apoptose responsable de la dénucléation a été inhibée chez ces animaux dont p53 est titrée par la protéine E6. En revanche, on note, au niveau des animaux $k: T$ des signes d'apoptose avec inhibition de la différenciation des fibres cristalliniemnes et, en certaines zones, prolifération inappropriée. Les animaux double-transgéniques développent des tumeurs du cristallin [3]. Dans cet exemple, comme dans le précédent, l'inactivation de $R b$ par E7 s'accompagne d'une augmentation de l'apoptose avec des signes de prolifération inappropriée alors que l'inactivation surajoutée de p53 par E6 permet le développement tumoral. Cette dernière anomalie isolée perturbe le phénomène d'apoptose qui joue un rôle important dans l'organogenèse terminale du cristallin.

[1. Kahn A, Briand P. médecine/sciences $1993 ; 9: 663-6$.]

[2. Howes KA, el al. (ienes I)ey 1994 ; $8: 1300-10.1$

[3. Pan H, Griep AE. Genes I)er $1994 ; 8: 1285-99$.] 